\title{
Determinación del polimorfismo de CYP2C9*2 y su relación con la farmacocinética de acenocumarol en voluntarios sanos.
}

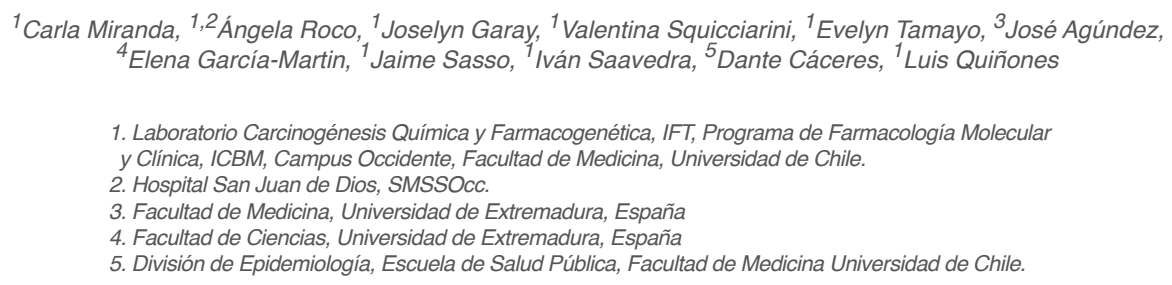

\section{Resumen:}

Antecedentes: La mayoría de los pacientes que reciben tratamientos con anticoagulantes orales por periodos prolongados presentan variabilidad en la respuesta. El acenocumarol es el anticoagulante oral más prescrito en nuestro país, es biotransformado principalmente por CYP2C9 e investigaciones recientes demuestran que la variante CYP2C $9 * 2$ es una de las responsables de la variabilidad de respuesta a acenocumarol.

Objetivo: Determinar las diferencias en los parámetros farmacocinéticos de acenocumarol en voluntarios que presentan la variante alélica CYP2C $9 * 2$.

Métodos: Se estudiaron 24 voluntarios sanos. La detección de genotipos se realizó mediante PCR-RFLP y los parámetros farmacocinéticos se obtuvieron mediante la concentración plasmática de acenocumarol usando un método validado para
UPLC-MS/MS.

Resultados: Del total de 24 voluntarios, 19 tenían el genotipo CYP2C9*1/*1 (wt/wt), 4 tenían genotipo CYP2C $9 * 1 / * 2$ (heterocigoto) y 1 voluntario tenía genotipo de CYP2C9*2/*2 (homocigoto recesivo). Los parámetros farmacocinéticos del acenocumarol no fueron significativamente diferentes entre los individuos con genotipo CYP2C $9 * 2$ y CYP2C $9 * 1$. Sin embargo, la farmacocinética de acenocumarol del individuo CYP2C $9 * 2 / * 2$ mostró diferencias relevantes con respecto a la observada en el grupo CYP2C $9 * 1 / * 1$ (tmáx aumentó 1,4 veces, ke disminuyó 1,8 veces y t1/2 aumentó 1,7 veces).

Conclusión: La farmacocinética de acenocumarol en el individuo con el genotipo CYP2C $9 * 2 / * 2$ refleja una potencial relevancia de este polimorfismo en el tratamiento con acenocumarol. 


\section{Relation of cy2c9*2 polymorphism and acenocumarol pharmacokynetics in healthy volunteers}

Background: Most of the patients receiving anticoagulant therapy for extended periods show variability in their clinical response. Acenocumarol, the most commonly prescribed oral anticoagulant in our country, is biotransformed mainly through CYP2C9 and recent research shows that $\mathrm{CYP} 2 \mathrm{C} 9 * 2$ variant is partly responsible for the variable response to acenocumarol.

Aim: to determine pharmacokinetics parameters of acenocumarol in volunteers exhibiting the CYP2C $9 * 2$ polymorphic variant.

Methods: Genotype detection was performed using PCR-RFLP and pharmacokinetics parameters were obtained from the acenocumarol concentrations, using a UPLC-MS/MS validated method. The project was approved by the institutional Ethics Committee of the University of Chile's Faculty of Medicine.

Results: 19 out of 24 volunteers had the CYP2C9*1/*1 genotype, 4 the $\mathrm{CYP} 2 \mathrm{C} 9 * 1 / * 2$ genotype (heterozygous) and 1 subject had the CYP2C $92 / * 2$ genotype (recessive homozygous). No statistically significant differences between acenocumarol pharmacokinetics parameters of CYP2C $9 * 2$ compared to those with normal variant, CYP2C9*1were observed.. However, a single individual with the CYP2C $9 * 2 / * 2$ genotype showed different pharmacokinetics parameters: tmáx and $\mathrm{t} 1 / 2$ were increased 1.4 and 1.7 times, respectively, and $\mathrm{kc}$ was 1.8 times lower compared to the group with the CYP2C $9 * 1 / * 1$ genotype.

Conclusion: There are clear differences in genotype-dependent acenocoumarol pharmacokinetics in individuals with the CYP2C $9 * 2 / 2$ genotype, reflecting a potential relevance of this polymorphism in anticoagulation with acenocumarol.

Keywords: Pharmacogenetics, CYP2C9*2, anticoagulants, acenocoumarol.

\section{Introducción}

En la experiencia clínica actual se sabe que, en general, los fármacos son eficaces sólo entre un 25 a un $60 \%$ de los pacientes, por lo tanto, es considerable el número de casos en los que se observa falla terapéutica o se presen$\tan$ reacciones adversas ${ }^{1-3}$. Esta variabilidad entre individuos es multifactorial, pero en la actualidad se conoce que la información predeterminada genéticamente es responsable entre un 25 a un $95 \%$ de la variabilidad de la respuesta a medicamentos, lo que es el foco principal de estudio de la Farmacogenética ${ }^{1-4}$, en especial para aquellos fármacos con una concentración plasmática mínima o un índice terapéutico estrecho, como son por ejemplo warfarina, acenocumarol o fenitoína ${ }^{1-3 ; 5 ; 6}$.
Las enzimas del citocromo P450 (CYP) que realizan la biotransformación de fármacos y otros xenobióticos pertenecen a tres familias CYP1, CYP2 y CYP3. Presentan en humanos un alto número de variables alélicas que podrían conducir a la supresión, disminución o aumento del metabolismo de fármacos. Por ende, es necesario conocer su variación en un individuo para ajustar a una terapia farmacológica individualizada, más eficaz y más segura ${ }^{7-9}$.

Entre las enzimas P450, CYP2C9 es una isoenzima que metaboliza aproximadamente el $15 \%$ de los medicamentos y que presenta polimorfismos genéticos que conducen a alteración de su actividad. El alelo silvestre es conocido como CYP2C $9 * 1$ y se denomina 
CYP2C9*2 cuando se produce un cambio nucleotídico o SNP (single nucleotide polymorphism) de una citosina por una timina en la posición 430 del gen (C430T) lo que conlleva a un cambio aminoacídico en la proteína de Arginina por Cisteína en la posición 144 de la secuencia de la enzima (rs1799853), esta mutación provoca que la variante $* 2$ presente alrededor de 8 veces menor actividad que la enzima normal 10;11. En poblaciones caucásicas se ha encontrado una frecuencia para esta variante de alrededor de un $19 \%$ para el genotipo heterocigoto (CYP2C9*1/*2) y un 3\% para el genotipo homocigoto recesivo $(\mathrm{CYP} 2 \mathrm{C} 9 * 2 / * 2){ }^{12 ; 13}$, similar a lo encontrado por nuestro grupo de investigación (12\% para *1/*2 y un $4 \%$ para $* 2 / * 2$ ) en 260 personas chilenas analizadas (datos no publicados).

En el tratamiento con anticoagulantes orales se observa una gran variabilidad en la respuesta debido a su estrecho margen terapéutico, una gran unión a proteínas plasmáticas (97\%), influencia de la dieta (consumo vitamina K) y a un gran número de interacciones farmacocinéticas y farmacodinámicas. Por esta razón es un área de vital importancia para individualizar la dosis diaria de cada paciente ${ }^{14}$.

Uno de los anticoagulantes orales más prescritos es el acenocumarol (Figura 1), el cual presenta mayor actividad que la warfarina ${ }^{14 ; 15}$. Se absorbe vía oral con rapidez, con una biodisponibilidad sistémica de un $60 \%$ como mínimo. Las enzimas involucradas en el metabolismo de acenocumarol son CYP2C9, CYP1A2 y CYP2C19 ${ }^{14}$. Investigaciones previas demuestran que CYP2C9 representa el 14\% de la variabilidad interindividual en la respuesta farmacológica ${ }^{14 ;}{ }^{15}$, por lo que no es posible establecer una correlación entre las concentraciones plasmáticas de acenocumarol y el nivel de protrombina o INR. Por lo tanto, determinar las variaciones genéticas interindividuales que explican las diferencias en la respuesta permitiría llevar un buen control de la terapia e indicar prescripción apropiada.

En Chile no existen estudios que hayan demostrado que la presencia de variaciones alélicas en CYP2C9 altere los parámetros farmacocinéticos de acenocumarol. De acuerdo a los antecedentes propuestos se busca implementar un método confiable, rápido y reproducible que permita la detección de la variante CYP2C9*2, el cual corresponde al polimorfismo con mayor relevancia clí- nica y además, relacionar la presencia de esta variante a cambios en los parámetros farmacocinéticos de acenocumarol en voluntarios sanos.

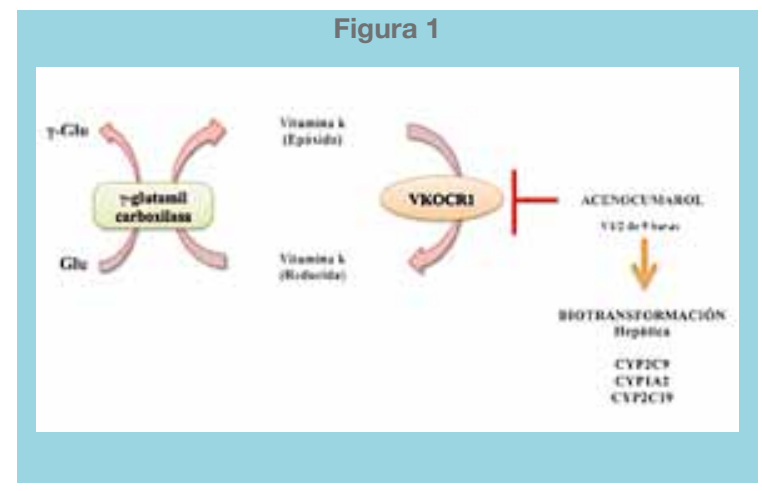

Mecanismo de acción y biotransformación de acenocumarol. VKOCR1: vitamina K epóxido reductasa.

\section{Materiales y Métodos}

Materiales: Como medicamento de estudio se utilizó comprimidos de $4 \mathrm{mg}$ de acenocumarol (Neo Sintrom®) de Laboratorio NOVARTIS. Los reactivos, solventes y material cromatográfico fueron adquiridos en Merck Química S. A. Las muestras de sangre se tomaron mediante una bránula ante braquial (Beckton \& Dickinson, $18 \mathrm{G}$ ).

En la técnica de PCR-RFLP se utilizaron partidores, dNTPs, Taq ADN Polimerasa (Invitrogen, Brasil), enzima de restricción AvalI (Eco47I) (Fermentas, USA), marcador de peso molecular 100-1000pb Hyperladder IV (Bioline, USA). La purificación del ADN de sangre periférica se realizó con un sistema comercial (Roche Diagnostics $\mathrm{GmbH}$, Alemania).

Voluntarios: Para el presente estudio, luego de la obtención de una autorización ética, los voluntarios fueron llamados vía página web del laboratorio (www.ift.cl), informados y consultados acerca de su voluntad de participar en el estudio, firmando un documento de consentimiento informado y autorizando una serie de exámenes para constatar su estado de salud. Estos exámenes incluyeron: examen físico completo, hemograma y VHS, tiempo de protrombina, orina completa, $\mathrm{VIH}$, hepatitis B y C, screening de drogas de abuso, glicemia, uremia, proteinemia, fosfatasas alcalinas, bilirubinemia, transaminasas oxálica 
y pirúvica y creatinemia. En el caso de las mujeres, se les practicó además un test de embarazo.

Se seleccionaron 24 voluntarios sanos (Tabla I) adultos originarios de Chile, hombres y mujeres de edades entre 18 y 55 años con apellidos hispanoamericanos y masa corporal (IMC) entre 19 y $30 \mathrm{Kgm} 2$, no fumadores, ni consumidores de drogas de abuso o alcohol, sin alergias a medicamentos y sin terapias concomitantes, además no debieron ingerir fármacos al menos dos meses antes del estudio. Finalmente, aquellos que presentaron rangos normales en los exámenes de laboratorio y después de previo examen físico (incluye la razón del índice normalizado de tiempo de protrombina (INR) entre 1 y 1,25), fueron declarados aptos para participar en el estudio.

\begin{tabular}{|c|c|c|c|}
\hline Genero & Mujeres & Hombres & Total \\
\hline Número & 13 & 11 & 24 \\
\hline Edad /años & $24,9 \pm 0,6$ & $24,1 \pm 0,3$ & $24,5 \pm 0,3$ \\
\hline Peso/ Kg & $60,8 \pm 0,6$ & $72,3 \pm 0,6$ & $66,1 \pm 0,3$ \\
\hline Altura / m & $1,598 \pm 0,003$ & $1,8 \pm 0,7$ & $1,678 \pm 0,004$ \\
\hline IMC / Kgm-2 & $23,8 \pm 0,2$ & $22,9 \pm 0,1$ & $23,39 \pm 0,08$ \\
\hline
\end{tabular}

Los voluntarios estuvieron en ayunas (mínimo 12 horas) e ingirieron una dosis oral de $4 \mathrm{mg}$ de acenocumarol con $250 \mathrm{~mL}$ de agua purificada. Las muestras de sangre periférica se recolectaron en tubos que contenían heparina sódica en los siguientes intervalos de tiempo: tiempo 0 (antes de administrar la droga), 0,$5 ; 1,0 ; 1,5 ; 2,0 ; 2,5 ; 3,0$; 3,$5 ; 4,0 ; 4,5 ; 5,0 ; 6,0 ; 8,0 ; 11,0$ y 24 horas post ingesta de acenocumarol. El plasma se obtuvo mediante centrifugación a 2.000 rpm en centrífuga refrigerada. Además, se recolectó una muestra en tubo que contenía EDTA para el análisis genotípico.

Los voluntarios estuvieron recluidos 14 horas en el centro y fueron atendidos por todo el equipo profesional, la alimentación fue diseñada por una nutricionista sobre la base de lo aconsejado por el médico. El médico llenó un formulario de reportes clínicos para cada voluntario que incluía una ficha de reacciones adversas a medicamentos
(RAM), exámenes clínicos y de laboratorio.

Aspectos éticos: El estudio fue aprobado por el Comité de Ética de Investigación en Seres Humanos de la Facultad de Medicina de la Universidad de Chile y fue desarrollado de acuerdo a las buenas prácticas clínicas y la declaración de Helsinki 16; 17 .

Determinación de CYP2C9*2 mediante PCR-RFLP: Se determinaron las variantes genéticas de CYP2C $9 * 2$ en los 24 voluntarios sanos mediante la técnica PCR-RLPutilizando una adaptación del método de Adithan et al, 2003 ${ }^{18}$, en el cual se utilizan los siguientes partidores de ADN: directo (5'-TACAAATACAATGAAAATATCATG-3') e inverso (5'-CTAACAACCAGACTCATAATG-3') ${ }^{19}$. Posteriormente, el amplicón obtenido fue sometido a digestión con la enzima de restricción AvaII.

Determinación cuantitativa de acenocumarol en plasma: Se validó el método de Huang et al, 2008 de Cromatografía Líquida de Ultra Resolución, acoplada a espectrometría de masa (UPLC-MS/MS) con modificaciones ${ }^{20}$. Se definió con respecto a la estabilidad, selectividad, sensibilidad, exactitud, precisión, recuperación, linealidad, reproducibilidad siguiendo la guía de FDA ${ }^{21}$.

El sistema cromatográfico consistió en un detector Quatro Micro API, Modelo ESCI Multimode-Ionization, Micromass y una unidad UPLC Acquity (Ultra Performance LC), un sistema Sample Manager and Binary Solvent Manager marca Waters. La columna utilizada fue una Acquity UPLC BEH HILIC $1.7 \mu \mathrm{m} 2.1$ x $50 \mathrm{~mm}$, Waters.

Farmacocinética: Los parámetros farmacocinéticos correspondientes a concentración plasmática máxima (Cmáx) y tiempo máximo (tmáx), área bajo la curva de concentraciones plasmáticas hasta 24 horas, $\mathrm{ABC}(0-\mathrm{t})$ y hasta tiempo infinito, $\mathrm{ABC}(0-\infty)$, tiempo de vida media $\left(t^{1} / 2\right)$ y constante de eliminación (ke), fueron determinados a partir de los datos obtenidos de las curvas de niveles plasmáticos de la droga en el tiempo post administración. Para ello se utilizó el procedimiento pk examine del paquete estadístico STATA 10.0, el cual es independiente de los modelos compartimentales [STATA 2007].

Análisis Estadísticos: Los cálculos de los parámetros fármacocinéticos se realizaron con el progama STATA, TX V10, 2007. Los análisis estadísticos se realizaron utilizando el programa GraphPad Prism versión 4.00 y el programa estadístico STATA 10.

Se aplicó una prueba de Shapiro Wilk a los datos de los 
parámetros farmacocinéticos, luego fueron comparados usando ANOVA de una vía con post test de Dunnett. Se aplicó una prueba paramétrica de modelo de regresión lineal $(\mathrm{Y}=\alpha+\beta 1$ gen $+\beta 2$ género $+\beta 3 \mathrm{imc}+\beta 4$ edad $+\mathrm{e})$. Los datos se reportaron como promedio y con un $95 \%$ de intervalo de confianza (CI 95\%).

\section{Resultados}

Los resultados obtenidos en el grupo de estudio mostraron que del total de 24 voluntarios, 19 poseen genotipo CYP2C9*1/*1 (wt/wt), 4 voluntarios poseen genotipo CYP2C $9 * 1 / * 2$ (heterocigoto) y 1 voluntario con genotipo de CYP2C9*2/*2 (homocigoto recesivo) (Figura 2).

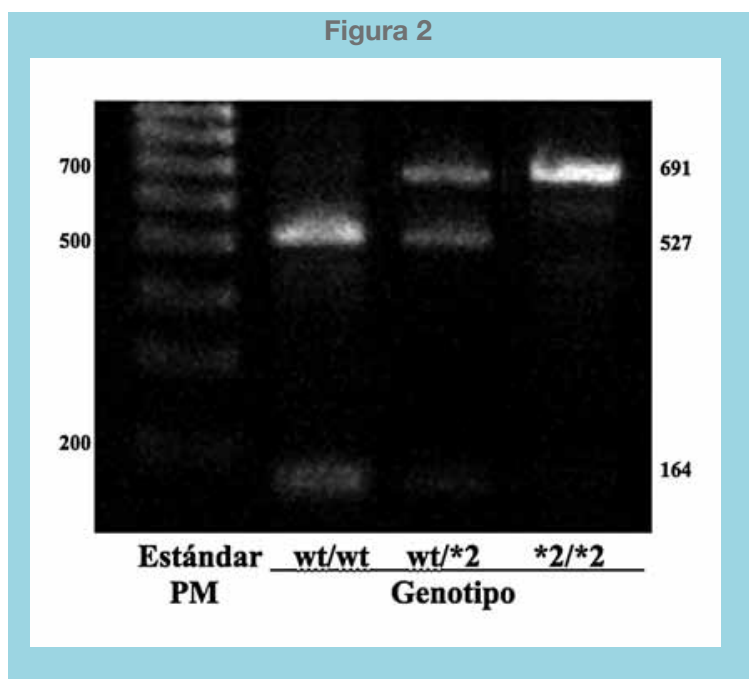

Patrón electroforético del polimorfismo genético estudiado. Después de la digestión del producto de la PCR con la enzima de restricción AvalI se observaron fragmentos de 527 y 164 pb para el genotipo silvestre $\left(C Y P 2 C 9^{*} 1 / * 1\right)$, de 691, 527 y 164 pb para el genotipo heterocigoto (CYP2C $9 * 1 / * 2)$ y 691 pb para el genotipo homocigoto mutado $($ CYP2C $9 * 2 / * 2)$.

Todos los voluntarios terminaron el estudio satisfactoriamente, de acuerdo con el protocolo. El análisis estadístico de comparación entre grupos de genotipos mostró que no se alcanza a observar diferencias estadísticamente significativas entre los diferentes grupos de individuos por genotipo y los parámetros farmacocinéticos estudiados.

Las variables antropométricas no influyeron mayormente esta evaluación, debido a que no existieron diferencias relevantes entre los grupos en cuanto a la edad y peso. El resultado de los parámetros farmacocinéticos estudiados (Cmáx, Tmáx, T1/2, ke o ABC) entre los diferentes grupos de genotipos se muestran en la Tabla II.

\begin{tabular}{|c|c|c|c|}
\hline $\begin{array}{l}\text { Parámetros } \\
\text { Farmacocinéticos }\end{array}$ & $*_{1} / *_{1}$ & $\underset{* 1 / * 2}{\text { CYP2C9 }}$ & $\star 2 / * 2$ \\
\hline $\mathbf{N}$ & 19 & 4 & 1 \\
\hline Cmáx / gmL-1 & $189 \pm 2$ & $178 \pm 10$ & 168 \\
\hline tmáx / h & $2,13 \pm 0,04$ & $2,25 \pm 0,02$ & 3 \\
\hline ke / h-1 & $0,069 \pm 0,001$ & $0,065 \pm 0,002$ & 0,038 \\
\hline$t^{1} 1 / 2 /$ horas & $10,8 \pm 0,2$ & $10,7 \pm 0,3$ & 18,5 \\
\hline ABC0à24 / g*hmL-1 & $1368 \pm 23$ & $1162 \pm 122$ & 1253 \\
\hline ABC0à» / $g^{*} h m L-1$ & $1736 \pm 32$ & $1522 \pm 180$ & 1887 \\
\hline
\end{tabular}

\section{Discusión}

Los anticoagulantes orales se utilizan para la prevención primaria y secundaria de eventos tromboembólicos. En Chile la política de salud establece como tratamiento administrar acenocumarol a personas de 15 años y más que presenten un accidente cerebrovascular isquémico (Guía GES, MINSAL), por lo que existe un amplio número de pacientes prescritos durante períodos prolongados con anticoagulantes antagonistas de vitamina $\mathrm{K}$.

La determinación de la dosis terapéutica apropiada de estos fármacos para los pacientes es compleja, esto hace que cobre interés determinar factores que puedan predisponer a estos individuos experimentar hemorrágicas severas. Por ello determinar las variaciones genéticas interindividuales que explican las diferencias en la respuesta a medicamentos, permitirá llevar un control de la terapia y una prescripción apropiada.

En este estudio no se encontraron diferencias estadísticamente significativas al comparar los parámetros farmacocinéticos de los voluntarios agrupados por genotipo, esto es, con genotipo CYP2C $9 * 2$ con respecto a los voluntarios con genotipo CYP2C9*1 (Figura 3 y 4). Esta falta de asociación estadística se debe en gran medida a que se encontró tan sólo 1 individuo con genotipo CYP2C9*2/*2 (doble mutado) en donde la manifestación de la falta de expresión de la enzima resultaría más evidente dado que en individuos CYP2C9*1/*2 (heterocigotos) la presencia del gen normal en uno de los alelos podría ser suficiente para que el organismo produzca la enzima necesaria y alcance así a metabolizar de manera semi-normal el acenocumarol. Ello se podría explicar debido a que el alelo silvestre se encuentra en un ambiente genético de activa transcripción (sin imprinting, represores, etc.) ${ }^{22}$. 


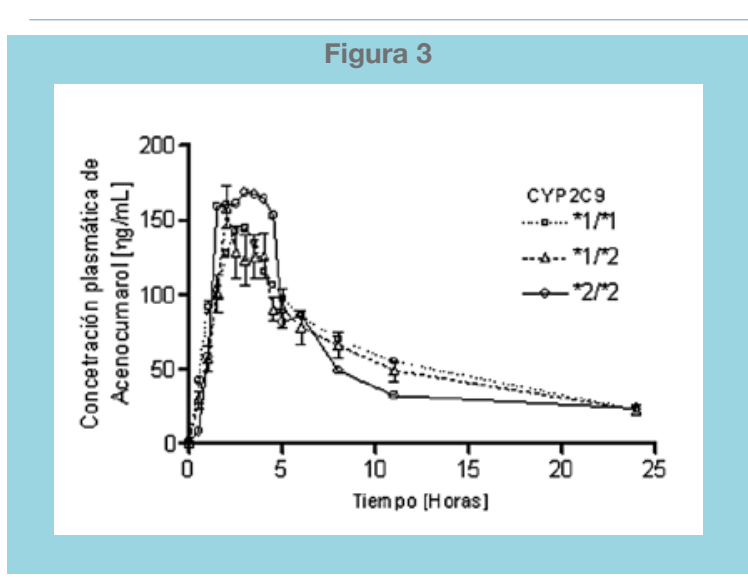

Curva farmacocinética promedio según genotipo para CYP2C9. Los datos están expresados como promedios \pm SEM.

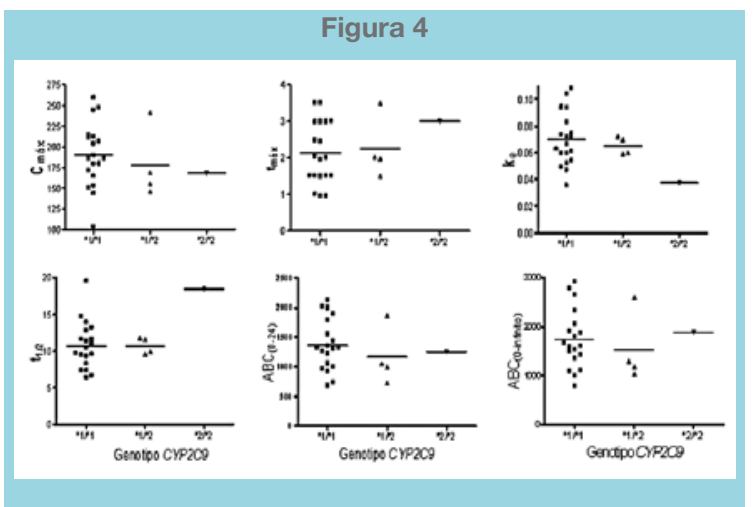

Gráficos de los parámetros farmacocinéticos de acenocumarol según su genotipo del voluntarios.
Sin perjuicio de lo anterior, la farmacogenética busca, a partir de la información genética de un individuo, predecir cómo éste va a responder frente a un tratamiento farmacológico, por lo tanto, el análisis individual cobra gran relevancia. En este aspecto, resulta muy interesante analizar el individuo que presenta genotipo CYP2C $9 * 2 / * 2$ de manera comparativa con respecto al promedio de las curvas farmacocinéticas de los voluntarios con genotipo CYP2C $9 * 1 / * 1$. La exclusiva presencia del alelo mutado del gen CYP2C9 (*2) modifica los parámetros farmacocinéticos, encontrándose que el tmáx se ve aumentado 1,4 veces en aproximadamente una hora, la ke se ve disminuida 1,8 veces y el t1/2 aumentado 1,7 veces. Esto sugiere una clara tendencia de menor eliminación del medicamento en este individuo con genotipo homocigoto mutado para CYP2C9*2. En concordancia con esto, estudios realizados por el grupo Benusiglio et al, en el 2007 encontraron que la dosis requerida de acenocumarol en personas con genotipo CYP2C9*1/*2 debe ser reducida en un 8 a $16 \%{ }^{23}$. Otro grupo encontró en 145 pacientes con genotipo CYP2C9*2 una dosis óptima de acenocumarol significativamente más baja que en los con genotipo CYP2C $9 * 1$ $(2,12 \pm 0.96 \mathrm{mg} / \mathrm{día}, \mathrm{p}<0,001)^{24}$. También se ha descrito que existe una mayor aparición de complicaciones hemorrágicas menores en los pacientes con el alelo $* 2$ que en otros pacientes sin la variable ( $\mathrm{p}<0,005, \mathrm{OR}=1,99$ [IC: 1,20 a 3,33] ${ }^{24 ; 25}$. Sin embargo, el grupo de Saraeva et al, en el 2007 no encontró una relación estadística al presentar un genotipo CYP2C $9 * 2{ }^{26}$.

De acuerdo a lo anterior, junto a los datos encontrados por nuestro grupo y frente a las investigaciones reportadas por otros grupos de investigación, es posible sugerir que el estudio de las variantes de CYP2C9 es de alta conveniencia para la terapia personalizada de acenocumarol en pacientes con genotipos mutados.

\section{Referencias:}

1. EVANS W, RELLING M. Pharmacogenomics: translating functional genomics into rational therapeutics. Science 1999; 286: 487-491.

2. INGELMAN-SUNDBERG M. Pharmacogenetics: an opportunity for a safer and more efficient pharmacotherapy. J Intern Med 2001; 250: 186-200.
3. SCHUETZ E. Induction of cytochromes P450. Curr drug Metab 2001; 2: 139-47.

4. PIRMOHAMED M, PARK B. Cytochrome P450 enzyme polymorphisms and adverse drug reactions. Toxicology 2003; 192: 23-32.

5. ROGERS J, NAFZIGER A, BERTINO J. Pharmacogenetics affects dosing, efficacy, and toxicity of cytochrome P450-metabolized drugs. Am J Med 2002; 113: 746-50. 
6. DALY A, KING B. Pharmacogenetics of oral anticoagulants. Pharmacogenetics 2003; 13: 247-252.

7. SMITH D, ABEL S, HYLAND R, JONES B. Human cytochrome P450s: selectivity and measurement in vivo. Xenobiotica 1998;28:1095-1128.

8. SHIMADA T, YAMAZAKI H, MIMURA M, INUI Y, GUENGERICH F. Interindividual variations in human liver cytochrome P-450 enzymes involved in the oxidation of drugs, carcinogens and toxic chemicals: studies with liver microsomes of 30 Japanese and 30 Caucasians. J Pharmacol Exp Ther 1994; 270 : 414-423.

9. CLARKE S. In vitro assessment of human cytochrome P450. Xenobiotica 1998; 28: 1167-1202.

10. AITHAL G, DAY C, KESTEVEN P, DALY A. Early report Association of polymorphisms in the cytochrome P450 CYP2C9 with warfarin dose requirement and risk of bleeding complications. Lancet 1999; 353: 717-719.

11. RETTIE A, WIENKERS L, GONZALEZ F, TRAGER W, KORZEKWA K. Impaired (S)-warfarin metabolism catalysed by the R144C allelic variant of CYP2C9. Pharmacogenetics 1994; 4: 39-42.

12. YASAR U, ELIASSON E, DAHL M, JOHANSSON I, INGELMAN-SUNDBERG M, SJÖQVIST F. Validation of methods for CYP2C9 genotyping: frequencies of mutant alleles in a Swedish population. Biochem Biophys Res Commun 1999; 254: 628-631.

13. GARCÍA-MARTÍN E, MARTÍNEZ C, LADERO J, GAMITO F, AGÚNDEZ J. High frequency of mutations related to impaired CYP2C9 metabolism in a Caucasian population. Eur J Clin Pharmacol 2001; 57: 47-49.

14. GADISSEUR A, VAN DER MEER F, ADRIAANSEN H, FIHN S, ROSENDAAL F. Therapeutic quality control of oral anticoagulant therapy comparing the short-acting acenocoumarol and the long-acting phenprocoumon. Br J Haematol 2002; 117: 940-946.

15. UFER M. Comparative pharmacokinetics of vitamin K antagonists: warfarin, phenprocoumon and acenocoumarol. Clin Pharmacokinet 2005; 44: 1227-1246.

16. Declaración de Helsinki de la Asociación Médica Mundial. Prin- cipios éticos para las investigaciones médicas en seres humanos. 2008.

17. Guidance for Industry. E6 Good Clinical Practice: Consolidated Guidance, U.S. Department of Health and Human Services, FDA, Center for Drug Evaluation and Research, ICH. 1996.

18. ADITHAN C, GERARD N, VASU S, BALAKRISHNAN R, SHASHINDRAN C, KRISHNAMOORTHY R. Allele and genotype frequency of CYP2C9 in Tamilnadu population. Eur J Clin Pharmacol 2003; 59: 707-709.

19. SULLIVAN-KLOSET, GHANAYEMB,BELLD,ZHANGZ, KAMINSKY L, SHENFIELD G, et al. The role of the CYP2C9 Leu359 allelic variant in the tolbutamide polymorphism. Pharmacogenetics 1996; 6: 341-349.

20. HUANG C, YANG J, DU Y, MIAO L. Measurement of free concentrations of highly protein-bound warfarin in plasma by ultra performance liquid chromatography-tandem mass spectrometry and its correlation with the international normalized ratio. Clin Chim Acta 2008; 393: 85-89.

21. Guidance for Industry. Bioanalytical Method Validation, U.S. Department of Health and Human Services, FDA, Center for Drug Evaluation and Research. 2001.

22. KUPIEC T, SHIMASAKI C. Pharmacists should be on the leading edge of "personalized medicine". America's Pharm 2010;20-24.

23. BENUSIGLIO P, DESMEULES J, DE MOERLOOSE P, DAYER P. Oral anticoagulation and pharmacogenetics: importance in the clinical setting. Revue médicale suisse 2007; 3: 2030, 2033-4, 2036.

24. MARK L, MARKI-ZAY J, FODOR L, HAJDARA I, PARAGH G, KATONA A. Cytochrome $\mathrm{P} 450$ 2C9 polymorphism and acenocoumarol therapy. Kardiol Pol 2006; 64: 397-402.

25. MÁRK L, MÁRKI-ZAY J, FODOR L, KONDACS A, PARAGH G, KATONA A. Significance of cytochrome P450 2C9 genotype for the bleeding complications in patients treated with acenocoumarol. Orv Hetil 2005; 146: 739-43.

26. SARAEVA R, PASKALEVA I, DONCHEVA E, EAP C, GANEV V. Pharmacogenetics of acenocoumarol: CYP2C9, CYP2C19, CYP1A2, CYP3A4, CYP3A5 and ABCB1 gene polymorphisms and dose requirements. J Clin Pharm Ther 2007; 32: 641-9. 